Comparison of the quick sequential organ failure assessment and systemic inflammatory response syndrome criteria for predicting sepsis in patients admitted to intensive care unit - study from a low-income setting

\title{
Ashani Ratnayake $^{(1)}$, Chamika Wanigasundara ${ }^{(2)}$
}

${ }^{1}$ Consultant Anaesthetist, District Base Hospital, Teldeniya, Sri Lanka, ${ }^{2}$ Senior registrar in Anaesthesia, Teaching Hospital, Peradeniya

\section{Background}

Sepsis is defined as life threatening organ dysfunction due to dysregulated host response to infection

In 2016, qSOFA replaced the traditional SIRS criteria in early identification of sepsis.

Manyl studies done in comparing these two scores in predicting sepsis in different setups .

Our primary objective this study was to determine the effectiveness of qSOFA score as a screening test in predicting sepsis in patients admitted to intensive care unit in a low income setting.

\section{Results}

Total number of 93 patients were studied.

Mean age $62.3 y r s$ with age range of 18 to 82 years $77.4 \%$ were male and $22.5 \%$ were female

$45.16 \%$ had no cormorbidities, $43.01 \%$ and $11.8 \%$ had less than 2

and more than 2 corrmorbidities respectively

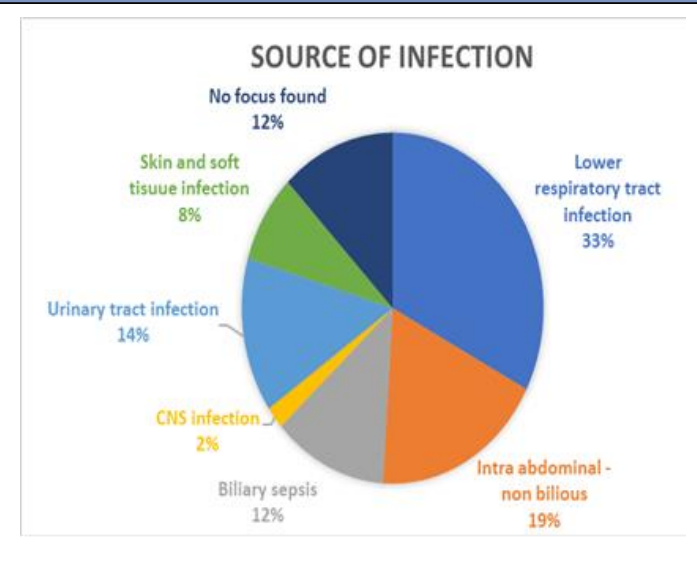

Screening tests need higher sensitivity

qSOFA has a sensitivity of $64.56 \%$, which carries a $45.5 \%$ of false negatives.

This is too high for a disease like sepsis with high mortality.

Literature suggest a screening test should have a sensitivity more than $80 \%$ to predict sepsis

\section{Method}

A prospective observational study conducted in the Medical and Surgical intensive care units of Teaching Hospital, Kandy - Sri Lanka.

The study period was from $1^{\text {st }}$ of March 2018 to $30^{\text {th }}$ of June 2018.

Study includes all patients admitted to ICU.

Patients under 18 years, pregnant patients, transfers from other ICUs and immediate post operative patients were excluded.

Demographic data, past medical history, clinical data required to calculate SIRS criteria and qSOFA were collected with a pre tested questionnaire.

Patients were followed upto see whether they develop sepsis. Data, samples taken in first 24 hours to avoid the possibility of unit acquired infection.

Confirmation of sepsis was done by positive cultures, radiological and surgical evidence and increment of SOFA score by 2 points.

Performance of qSOFA and SIRS criteria was compared using measurement of sensitivity, specificity and positive and negative predictive value.

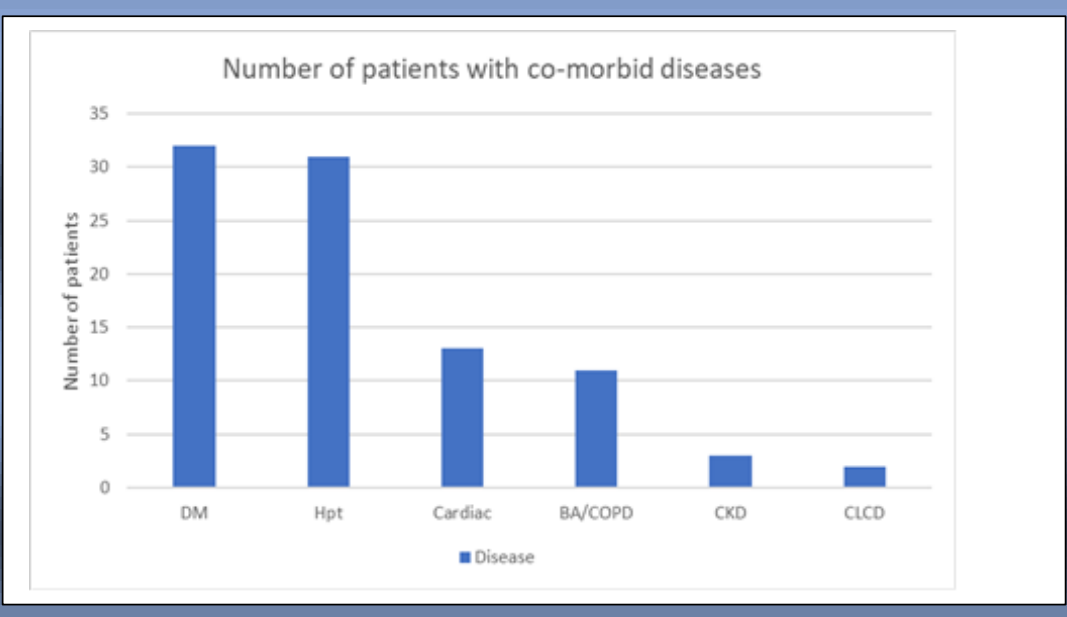

Performance of qSOFA and SIRS criteria in predicting sepsis

\begin{tabular}{|c|c|c|c|}
\hline & Sepsis & $\begin{array}{l}\text { No } \\
\text { sepsis }\end{array}$ & value \\
\hline $\begin{array}{l}\text { qSOFA } \\
+\end{array}$ & 31 & 8 & \multirow{2}{*}{$\begin{array}{l}\text { Sensitivity }=64.56 \% \\
\text { Specificity }=82.22 \% \\
\text { PPV } \quad=71.48 \% \\
\text { NPV } \quad=68.51 \%\end{array}$} \\
\hline qSOFA & 17 & 37 & \\
\hline SIRS+ & 38 & 33 & \multirow{2}{*}{$\begin{aligned} \text { Sensitivity } & =79.6 \% \\
\text { Specificity } & =26.665 \\
\text { PPV } & =53.33 \% \\
\text { NPV } & =79.48 \%\end{aligned}$} \\
\hline SIRS - & 10 & 12 & \\
\hline
\end{tabular}

\section{Conclusion :}

Predictive scoring systems are used in its diagnosis of sepsis. qSOFA replaced SIRS criteria as a screening test for sepsis. Our study does not support qSOFA as a better screening tool for sepsis. 